\title{
Genetic Programming Approach for Positron Collisions with Alkali-metal Atom
}

\author{
Amr Radi \\ Lecture \\ Physics Department, Faculty of Science \\ Ain Shams University, Cairo, Egypt \\ 0020101436582 \\ A.Radi@asunet.shams.edu.eg
}

\author{
Salah Yaseen El-Bakry \\ Physics Department, Faculty of Science \\ Taibah University, Madinah Munawwarah, \\ P.O.Box 344, Kingdom of Saudi Arabia \\ 00966500071670 \\ elbakry_salah@Yahoo.com
}

\begin{abstract}
Genetic Programming (GP) has been used to discover the function that describes the collisions of positrons with sodium, potassium, rubidium and cesium atoms at low and intermediate energies. The discovered function shows a good match to the experimental data. We find that the GP technique is able to improve upon more traditional methods.
\end{abstract}

\section{Categories and Subject Descriptors}

H.1 [Information Systems]: Models and Principles.

Subject Descriptors: G.4 and D.4.0

\section{General Terms}

Experimentation and Verification.

\section{Keywords}

Genetic Programming, positron collisions, alkali-metal atoms and total collisional cross sections.

\section{INTRODUCTION}

From the theoretical atomic collision point of view, the interest of many authors in positron-atom collisions (say positron-alkali atom scattering) was concentrated until now on the study of the various scattering channels (i.e. elastic, positronium formation, atomic excitation and ionization ) through the calculation of the scattering parameters such as phase shifts, scattering lengths and cross sections using different conventional methods for low, intermediate and high energy regions ( see e.g. Refs.[1,2] ).. ElBakry and Radi[2] presented a new technique for approach for electron-alkali-metal atom collisions. This approach shows a good match to the experimental data and a better performance over other theoretical calculations.

In the present work, GP is fed with incident energies $\varepsilon$ and atomic number of the alkali target atom $z$ so that the outputs imitate the experimental data.

Our representation, the fitness function is calculated as a negative value of the total absolute performance error of the discovered function on the experimental data set, i.e. a lower error must correspond to a higher fitness [3]. The running process stops when the error $\mathrm{E}$ is reduced to an acceptable level.

Copyright is held by A.Radi \& S. El Bakry

GECCO'07, July 7-11, 2007, London, England, United Kingdom

ACM 978-1-59593-697-4/07/0007.
The training data set which is used based on experimental data for the total scattering cross sections of the collisions of positrons with $\mathrm{Na}$, $\mathrm{K}, \mathrm{Rb}$ and $\mathrm{Cs}$ atoms $[2,4]$.

The final discovered function $\sigma(\varepsilon, z)$ for describing the electron collisions with alkali atoms at low and intermediate energies

\section{RESULTS AND CONCLUSION}

Our discovered function was tested using the experimental data of the total collisional cross sections (measured in $\pi a_{0}^{2}$ units). The training data is based on experimental observations at incident energies.

A good match between the experimental data of the total collisional cross sections and the calculated ones by employing our discovered function had been found. Then, the discovered function has been used to predict cross sections of $\mathrm{e}^{+}-\mathrm{Cs}$ scattering, at incident energies ranging from $0.7 \mathrm{eV}$ to $60 \mathrm{eV}$ which corresponds to the available experimental data for $\mathrm{e}^{+}-\mathrm{Cs}$ scattering [4]. A best fitting between the experimental and predicted total collisional cross sections using our discovered function..

Finally, we conclude that GP has become one of important research areas in the field of atomic collision physics. The present work presents a new technique for modeling the total collisional cross sections of positrons with sodium, potassium, rubidium and cesium alkali atoms based on GP technique. The discovered function shows a good match to the experimental data.

\section{REFERENCES}

[1] David D Reid and J M Wadehra, Phys.Rev.A57 2583 (1998).

[2] S El-Bakry and Amr Radi, Int. J. Mod. Phys. B, vol.20, No.32 (2006).

[3] J. Koza, M. Keane, M. Streeter, W. Mydlowec, J. Yu, Genetic Programming IV: Routine Human-Competitive Machine Intelligence, Guido Lanza - Computers - (2005).

[4] E W McDaniel, Atomic collisions: Electron and photon projectiles (Wiley, New York, 1989). 